Supplement of Geosci. Model Dev., 9, 2143-2151, 2016

http://www.geosci-model-dev.net/9/2143/2016/

doi:10.5194/gmd-9-2143-2016-supplement

(C) Author(s) 2016. CC Attribution 3.0 License.

(c) (i)

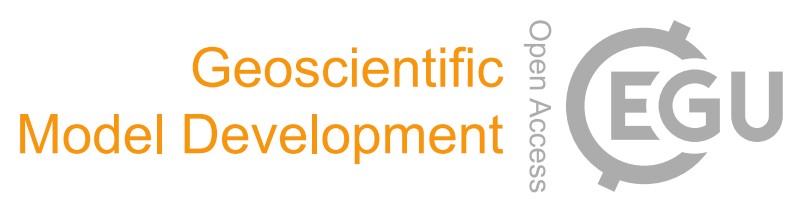

Supplement of

\title{
Development of aroCACM/MPMPO 1.0: a model to simulate secondary organic aerosol from aromatic precursors in regional models
}

Matthew L. Dawson et al.

Correspondence to: Donald Dabdub (ddabdub@uci.edu)

The copyright of individual parts of the supplement might differ from the CC-BY 3.0 licence. 
Table S1. Component species and surrogate structures of SOA groups in the original and updated CIT model. Surrogate species are indicated with a $\left(^{*}\right)$.

\section{Original}

\begin{tabular}{lll}
$\begin{array}{lll}\text { SOA } \\
\text { Group }\end{array}$ & $\begin{array}{l}\text { CIT } \\
\text { Model }\end{array}$ & $\begin{array}{l}\text { aroCACM } \\
\text { MPMPO }\end{array}$ \\
\hline A1 & UR21 & UR21* \\
& UR28* & UR28 \\
& & \\
A2 & RP13 & RP13 \\
& RP17 & RP17 \\
& RP18* & RP18 \\
& UR29 & UR29 \\
& UR30 & UR30 \\
& UR26 & UR3*
\end{tabular}

A3 RPR9* RPR9*

RP12 RP12

A4 UR3* UR26*

UR23 UR23

UR8 UR8

A5 UR17* UR17*

UR7 UR7

B1

$\begin{array}{ll}\text { AP1 } & \text { AP1 } \\ \text { AP6* } & \text { AP6* } \\ \text { UR31 } & \text { UR31 }\end{array}$

B2

$\begin{array}{ll}\text { ADAC } & \text { ADAC } \\ \text { RPR7* } & \text { RPR7* } \\ \text { RP14 } & \text { RP14 } \\ \text { RP19 } & \text { RP19 } \\ \text { UR2 } & \text { UR2 } \\ \text { UR14 } & \text { UR14 } \\ \text { UR27 } & \text { UR27 }\end{array}$

B3

AP10* AP10*

UR11 UR11

UR15 UR15
Original

\begin{tabular}{lll}
$\begin{array}{l}\text { SOA } \\
\text { Group }\end{array}$ & $\begin{array}{l}\text { CIT } \\
\text { Model }\end{array}$ & $\begin{array}{l}\text { aroCACM/ } \\
\text { MPMPO }\end{array}$ \\
\hline B4 & AP11 & AP12* \\
& AP12* & \\
& UR20 & \\
& UR34 & \\
& & \\
B5 & AP8 & AP8 \\
& UR5 & UR5 \\
& UR6 & UR6 \\
& AP7* & AP7*
\end{tabular}

B6

AP11*

B7

UR20*

C1

UR75*

UR24

UR72

C2 UR77*

RP98

RPR4

UR22

UR66

UR57

UR58

UR65

C3

PN11*

PN12

PN13

PN14

C4

R102*

UR76

C5
RP30*

RP29

RP31 
Table S2. Domain and 24 hour average concentrations of aromatic-derived lumped SOA species C1 - C5 in the 30\% each $m$-xylene and toluene scenario.

\begin{tabular}{lcc} 
& $\begin{array}{c}\text { Average } \\
(\mu \mathrm{g} / \mathrm{m} 3)\end{array}$ & $\begin{array}{c}\text { S Standard } \\
\text { Deviation } \\
\left(\mu \mathrm{g} / \mathrm{m}^{3}\right)\end{array}$ \\
\hline C1 & $2.36 \mathrm{E}-01$ & $4.24 \mathrm{E}-01$ \\
C2 & $3.54 \mathrm{E}-02$ & $6.18 \mathrm{E}-02$ \\
C3 & $1.03 \mathrm{E}-01$ & $1.30 \mathrm{E}-01$ \\
C4 & $9.42 \mathrm{E}-03$ & $1.88 \mathrm{E}-02$ \\
C5 & $1.55 \mathrm{E}-02$ & $2.00 \mathrm{E}-02$
\end{tabular}

